\title{
Universal canonical black hole entropy
}

\author{
Ashok Chatterjee* and Parthasarathi Majumdar ${ }^{\dagger}$ \\ Theory Group, Saha Institute of Nuclear Physics, Kolkata 700 064, India.
}

\begin{abstract}
Non-rotating black holes in three and four dimensions are shown to possess a canonical entropy obeying the Bekenstein-Hawking area law together with a leading correction (for large horizon areas) given by the logarithm of the area with a universal finite negative coefficient, provided one assumes that the quantum black hole mass spectrum has a power law relation with the quantum area spectrum found in Non-perturbative Canonical Quantum General Relativity. The thermal instability associated with asymptotically flat black holes appears in the appropriate domain for the index characterising this power law relation, where the canonical entropy (free energy) is seen to turn complex.
\end{abstract}

The microcanonical entropy $\left(S_{M C}\right)$ of generic stationary non-rotating macroscopic four dimensional black holes, modelled as isolated horizons [1], has been shown to obey, for large fixed horizon areas, the Bekenstein-Hawking Area Law (BHAL) [2] together with an infinite series of corrections, each term of which is finite and calculable [3]. The leading correction is logarithmic in area (BH entropy) with a coefficient (calculated in [3]) that has been argued to be universal [4], [5]. These results are obtained within Non-perturbative Canonical Quantum General Relativity (NCQGR), and crucially depend on the discrete spectrum of geometrical observables, especially the area [6].

It is well-known in elementary statistical mechanics [7] that, unlike the canonical entropy, the definition of the microcanonical entropy in fact is not unique. One can define it as the logarithm of the degeneracy of quantum microstates characterizing the system, as has been employed in the calculations cited above [2], [3]. Alternatively, one can define it as the logarithm of the density of these microstates as a function of the energy. While both these definitions yield the same area law, they donot lead to the same logarithmic corrections. The difference actually depends on the relation between the energy (mass) of the black hole and the horizon area. This difference plays an important role when the NCQGR result is used to compute the effect of thermal fluctuations on the canonical entropy of black holes [8],

In [8], the precise distinction and relationship between corrections to the BHAL arising due to quantum spacetime fluctuations, and due to thermal fluctuations within a canonical framework (admittedly heuristic), has been delineated. The logarithmic corrections due to quantum spacetime fluctuations are interpreted as 'finite size effects'. ${ }^{1}$ Thermal fluctuations then generate additional log-area corrections which are computed in [8] for generic non-rotating antide Sitter black holes. Fortunately, the thermal fluctuation contributions to the canonical entropy can be expressed entirely in terms of the microcanonical entropy which has been computed within NCQGR as already mentioned. Unfortunately, however, the extra logarithmic contribution to the canonical entropy owing to the difference in the two definitions of the microcanonical entropy has been missed out in [8].

Note that within NCQGR the spectrum of the observable corresponding to the classical isolated horizon mass is not known, while the discrete spectrum of the area observable is completely understood [6]. Consequently, the difference between the two microcanonical entropy definitions cannot actually be computed within NCQGR. This also implies that the canonical ensemble cannot be defined in the NCQGR formalism with as much rigour and reliability as the microcanonical ensemble.

In this paper, we postulate that the two observables (mass and area) have a power-law relationship for large mass (area). We then proceed heuristically to define a canonical partition function in the standard manner. Restricting to macroscopic black holes of large area (energy), this partition function is computed using a saddle-point approximation and the canonical entropy extracted from it. The difference in the two definitions of microcanonical entropy now manifests as an extra logarithmic correction to the BHAL for the canonical entropy, over and above logarithmic terms arising from quantum spacetime fluctuations [3] and thermal fluctuations [11], [8], [12] (for adS black holes). One observes that, just like the earlier microcanonical entropy result [3], the complete canonical entropy also has a logarithmic correction to the BHAL with a universal coefficient, independent of the index characterizing the assumed

\footnotetext{
*email: ashok@theory.saha.ernet.in

${ }^{\dagger}$ On deputation from the Institute of Mathematical Sciences, Chennai 600 113, India; email: partha@theory.saha.ernet.in

${ }^{1}$ Computations of the entropy for matter fields in fixed Euclidean classical black hole backgrounds have been performed in [9]. Our approach differs from these in that we focus on the non-perturbative quantum fluctuations of the Lorentzian black hole spacetime itself. This is also in contrast to the work of ref. [10] which is purely perturbative in nature.
} 
power-law relation between the mass and the area. However, the value of the index plays a crucial role in designating the domain of validity of the assumption of thermal equilibrium, and hence of the entire computation.

We should mention that for specific adS black holes, the extra logarithmic correction to the canonical entropy has also been suggested in [12] (as a kind of 'Jacobian' factor), upon the assumption of an equally-spaced area spectrum. ${ }^{2}$ In addition, a power-law relation between the area and mass spectra has been assumed, as here. The extra logarithmic correction to the canonical entropy has been computed for specific adS black holes in [12], and the results appear to be in agreement with our general formulation. The microcanonical entropy contribution has of course not been considered at all in [12]. Thus, the actual source of the extra log terms has not been traced in [12] to the difference in the two definitions of microcanonical entropy, as we have done here.

The canonical partition function is given, as always, by

$$
Z_{C}(\beta)=\operatorname{Tr} \exp -\beta \hat{H} .
$$

where, as argued in section IV of [8], only the part of the Hamiltonian pertaining to the (inner) boundary of spacetimeis relevant for thermodynamics, the bulk part being rendered redundant by the quantum Hamiltonian constraint. Taken together with caveats mentioned above, one can heuristically reexpress eq. (1) as a sum

$$
Z_{C}=\sum_{p} g\left(E\left(A_{p}\right)\right) \exp -\beta E\left(A_{p}\right)
$$

where, $A_{p}$ is the allowed area of a very large black hole horizon with $p,(p \gg 1)$ punctures due to the impinging of spin network edges on the horizon; each puncture carries a spin $1 / 2$. The area spectrum, as given by the NCQGR formalism then implies that $A_{p} \sim p$. We further assume that the energy (mass) of the black hole is a monotonic function of the area eigenvalue. $g\left(E\left(A_{p}\right)\right)$ is the degeneracy of the energy level designated by $E\left(A_{p}\right)$. It is important to keep in mind that eq. (2) is actually valid only for very large (average) areas, i.e., for large (average) energies, since only in the limit of large mass or areas do black holes ever have a chance of being in thermal equilibrium with radiation. Thus the sum (2) is dominated by large values of $p \gg 1$. Using now the Poisson resummation formula

$$
\sum_{n=-\infty}^{\infty} f(n)=\sum_{m=-\infty}^{\infty} \int_{-\infty}^{\infty} d x \exp (-2 \pi i m x) f(x)
$$

one obtains

$$
Z_{C} \simeq \int_{-\infty}^{\infty} d x g(E(A(x))) \exp -\beta E(A(x))
$$

where, we have set $\sum_{l} \exp -2 \pi i l x \approx 1$ for $|x| \gg 1$, as appropriate to very large black holes. Changing integration variables from $x$ to $E$ we obtain,

$$
\begin{aligned}
Z_{C} & =\int d E\left(\frac{d E}{d x}\right)^{-1} g(E) \exp -\beta E \\
& =\int d E \exp \left[S_{M C}(E)-\log \left|\frac{d E}{d x}\right|-\beta E\right] .
\end{aligned}
$$

In eq. (5), the microcanonical entropy $S_{M C} \equiv \log g(E)$ as used in [2] and [3] to calculate the entropy of non-rotating isolated horizons; this is not the definition used in [8] where instead we identified the logarithm of the density of states as the microcanonical entropy. In doing so, the second term in the exponent above, namely $-\log \left|\frac{d E}{d x}\right|$ has been missed in [8]. In other words, defining $\tilde{S}_{M C} \equiv \log \rho(E)$, the two definitions of microcanonical entropy are related by

$$
\tilde{S}_{M C}(E)=S_{M C}(E)-\log \left|\frac{d E}{d x}\right| .
$$

Obviously, the extra contribution is irrelevant for demonstrating the BHAL, but since we are interested in ascertaining log-area corrections to the BHAL for canonical entropy, the effect of the second term on the rhs above must be investigated.

\footnotetext{
${ }^{2}$ This assumption, without the underpinning of a theory of quantum spacetime fluctuations as here, is in fact adhoc.
} 
We proceed as in [8] by evaluating the integral in a saddle-point approximation to yield, for the saddle-point $E=M$

$$
\left.Z_{C} \simeq \exp \left\{S_{M C}(M)-\beta M-\log \left|\frac{d E}{d x}\right|_{E=M}\right]\right\}\left[\frac{\pi}{-S_{M C}^{\prime \prime}(M)}\right]^{1 / 2}
$$

The canonical entropy is now straightforward to extract, using the standard thermodynamic formula $S_{C}=\log Z_{C}+$ $\beta M$, leading to

$$
\begin{aligned}
S_{C} & =S_{M C}(M)-\log \left|\frac{d E}{d x}\right|_{E=M}-\frac{1}{2} \log \left[-S_{M C}^{\prime \prime}(M)\right]+\text { const. } \\
& =S_{M C}(M)-\frac{1}{2} \log (-\Delta)
\end{aligned}
$$

where,

$$
\left.\Delta \equiv \frac{d^{2} S_{M C}}{d E^{2}}\left(\frac{d E}{d x}\right)^{2}\right|_{E=M}
$$

Manipulating the derivatives in eq. (9) using the chain rule several times, it now follows that

$$
\Delta=\left.\left[\frac{d^{2} S_{M C}}{d A^{2}}-\left(\frac{d S_{M C}}{d A}\right) \frac{d^{2} E / d A^{2}}{d E / d A}\right]\left(\frac{d A}{d x}\right)^{2}\right|_{E=M} .
$$

Since $S_{M C}$, computed within NCQGR as a function of the horizon area ( [2], [3]) contains universal logarithmic corrections [3], [4], and the area spectrum for large isolated horizons is known to be linear as a function of $x$ [6], essentially the only 'nonuniversal' aspect of the canonical entropy above is the second term within the square brackets which involves the energy (mass)-area relation. We now hypothsize that this relation is a power law one (for large area),

$$
E(A)=\text { const. } A^{r}
$$

where, $r$ is a real number, positive or negative. This relation, together with the formula for microcanonical entropy $[3]$

$$
S_{M C}=S_{B H}-\frac{3}{2} \log S_{B H}+\text { const. }+O\left(S_{B H}^{-1}\right)
$$

yields the canonical entropy given by the BHAL and universal logarithmic corrections,

$$
S_{C}=S_{B H}-\log S_{B H}-\frac{1}{2} \log (r-1)+\text { const. }+O\left(A^{-1}\right) .
$$

It is of interest to note that the leading logarithmic correction is actually independent of the exponent $r$ characterizing the mass-area relation, which is different for different classes of black holes. However, this exponent plays a most crucial role: it decides whether the black hole is in thermal equilibrium or not. E.g., for asymptotically flat non-rotating black holes of large macroscopic horizon size, $r=1 / 2<1$. This implies that the canonical entropy (and the Gibbs free energy) has turned complex for such black holes, clearly signifying a thermal instability. The saddle-point approximation used to extract the canonical entropy breaks down whenever $r<1$.

On the other hand, all generic non-rotating anti-de Sitter black holes have $r>1$ in the parameter domain characterized by a large fixed cosmological constant. This is basically the domain in which the horizon radius is far larger than the length parameter $\ell \sim \Lambda^{-1 / 2}$. Thus, for the non-rotating BTZ black hole, $r=2$ while for the adS-Schwarzschild black hole in four dimensions, $r=3 / 2$, so that thermal equilibrium can indeed ensue in this domain of parameter space. Incorporation of electric charge seems to involve no extra complication generically. As the exponent $r \rightarrow 1$ from above, for the adS black holes one appears to approach the Hawking-Page phase transition [14] from the black hole 'phase' to an adS 'gas phase'.

Consider now an alternative approach to the canonical entropy, based not on a canonical energy ensemble, but rather a canonical area ensemble [15]. This is motivated by the observation already made, viz., the mass of the horizon is not a well-defined observable in NCQGR, while the area is. It is for this reason that the microcanonical entropy within this formalism automatically yields a result as a function of the horizon area, without any reference to the spectrum of any energy operator. To define a canonical partition function in such an area ensemble, we shall 
have to require that the total area (just like the total energy in the standard canonical ensemble) is a constant of motion. To achieve this, we may think of area fluctuations being caused by the addition or deletion of deficit angles made on the horizon 2-sphere by the appearance of new spin network edges impinging on the horizon, or old edges disappearing, by a yet unknown mechanism. All these fluctuations keep a 'total' area fixed.

The partition function for such an area ensemble is given by [15]

$$
\begin{aligned}
Z(\alpha) & =\operatorname{Tr} \exp -\alpha \hat{A} \\
& =\sum_{p} g\left(A_{p}\right) \exp -\alpha A_{p},
\end{aligned}
$$

where $\alpha$ is the corresponding inverse ambient 'temperature'. ${ }^{3}$ The relation of $\alpha$ to the inverse temperature $\beta$ in a standard canonical ensemble is far from clear. $p$ is the number of punctures; we assume as before that in the formula (14) above, the rhs is dominated by terms of large $A_{p}$, assumed to be linearly dependent on large $p$. Appealing to the Poisson resummation formula as before, the partition function can be rewritten as an integral over area

$$
Z(\alpha)=\int d A \exp \left\{S_{M C}(A)-\log \left|\frac{d A}{d x}\right|-\alpha A\right\}
$$

The integral can be evaluated as before by a saddle point approximation, yielding,

$$
Z(\alpha)=\exp \left\{S_{M C}\left(A_{0}\right)-\log \left|\frac{d A}{d x}\right|_{A=A_{0}}-\alpha A_{0}\right\}\left[\frac{\pi}{-S_{M C}^{\prime \prime}\left(A_{0}\right)}\right]^{1 / 2}
$$

where $A_{0}$ is the saddle point (equilibrium) area. Observe at this point that, given that the area spectrum is linear in $x$ for large $x$, the $|d A / d x|^{-1}$ term in the integral is a constant independent of the area. This is quite unlike the earlier situation in the standard canonical ensemble where this particular Jacobian term had nontrivial implications for the canonical entropy. Furthermore, the last factor in eq, (16), representing the contribution due to thermal fluctuations, is once again completely determined by the microcanonical entropy calculated within NCQGR.

However, observe that using eq. (12) one immediately gets $S_{M C}^{\prime \prime}\left(A_{0}\right)>0$. It follows that within the canonical area ensemble, it is not possible to use the saddle-point approximation to calculate the effect of Gaussian thermal fluctuations on logarithmic corrections to the canonical entropy of all general relativistic black holes. The thermal instability discerned in the standard 'energy' canonical ensemble for asymptotically flat black holes now appears universally for this area ensemble, This raises the question as to whether the area ensemble is at all an equilibrium thermodynamic ensemble for black holes of large area.

In conclusion, we reiterate that the universality of the logarithmic correction found by us in the standard canonical ensemble, emerges without any information about the actual mass spectrum of the black hole as a function of the area. It is enough to assume the mere existence of a power law relation. Furthermore, no additional assumption at all needs to be made regarding the nature of the area spectrum which is completely determined within the NCQGR formalism [6]. The fact that the exponent characterizing the power law area-mass relation is decisive in delineating the domain of thermal equilibrium vis-a-vis black holes is also an interesting, albeit an expected, feature. It is however, extremely important to keep in mind the caveat in all of the above, of extending a classical relation between mass and area of black holes to the spectra of the corresponding quantum operators. Elimination or otherwise of this caveat remains the top priority issue in future work on this problem.

[1] A. Ashtekar, C. Beetle and S. Fairhurst, Class. Quant. Grav. 17 (2000) 253; A. Ashtekar, C. Beetle and J. Lewandowski, Class. Quant. Grav. 19 (2000) 1195.

[2] A. Ashtekar, J. Baez and K. Krasnov, Adv. Theor. Math. Phys. 4 (2000) 1 and references therein.

[3] R. Kaul and P. Majumdar, Phys. Rev. Lett. 84 (2000) 5255; P. Majumdar, Pramana 55 (2000) 511 and Black hole entropy: classical and quantum aspects, hep-th/0110198.

\footnotetext{
${ }^{3}$ Brown et. al. [13] have referred to a quantity analogous to $\alpha$, i.e., the canonical conjugate to area, as the 'surface pressure'.
} 
[4] S. Carlip, Class. Quant. Grav. 7 (2000) 4175.

[5] T. R. Govindarajan, R. Kaul and S. Varadarajan, Class. Quant. Grav. 18 (2001) 2877.

[6] A. Ashtekar and J. Lewandowski, Class. Quant. Grav. 14 (1997) 55.

[7] K. Huang, Statistical Mechanics,John Wiley and Sons (1963).

[8] A. Chatterjee and P. Majumdar, Black hole entropy: quantum vs thermal fluctuations, gr-qc/0303030.

[9] R. B. Mann and S. Solodukhin, Phys. Rev. D55, 3622 (1997); D. Binosi, V. Moretti, L. Vanzo and S. Zerbini, ibid. D59, 104017 (1999); A. Medved and G. Kunstatter ibid. D60, 104029 (1999).

[10] A. A. Bytsenko, L. Vanzo and S. Zerbini, Phys. Rev. D57, 4917 (1998).

[11] S. Das, P. Majumdar and R. Bhaduri, Class. Quant. Grav. 19 (2002) 2355.

[12] G. Gour and A.J.M. Medved, Class. Quant. Grav. 20 (2003) 3307.

[13] J. Brown, G. Gomer, E. Martinez, J. Melmed, B. Whiting and J York, Jr., Class. Quant. Grav. 7 (1990) 1433.

[14] S. W. Hawking and D. N. Page, Commun. Math. Phys. 87 (1983) 577.

[15] K. Krasnov, Class. Quant. Grav. 16 (1999) 563. 